\title{
DYNAMIC STABILITY OF FUNCTIONALLY GRADED PLATE UNDER IN-PLANE COMPRESSION
}

\author{
ANDRZEJ TYLIKOWSKI
}

Received 2 March 2004

Functionally graded materials have gained considerable attention in the high-temperature applications. A study of parametric vibrations of functionally graded plates subjected to in-plane time-dependent forces is presented. Moderately large deflection equations taking into account a coupling of in-plane and transverse motions are used. Material properties are graded in the thickness direction of the plate according to volume fraction power law distribution. An oscillating temperature causes generation of in-plane timedependent forces destabilizing the plane state of the plate equilibrium. The asymptotic stability and almost-sure asymptotic stability criteria involving a damping coefficient and loading parameters are derived using Liapunov's direct method. Effects of power law exponent on the stability domains are studied.

\section{Introduction}

Functionally graded materials have gained considerable attention in the high-temperature applications. Functionally graded materials are composite materials, which are microscopically inhomogeneous, and the mechanical properties vary smoothly or continuously from one surface to the other. It is this continuous change that results in gradient properties in functionally graded materials (FGM). Commonly, these materials are made from a mixture of ceramic and metal or a combination of different metals. The ceramic material provides high temperature resistance due to its low thermal conductivity while the ductile metal component prevents fracture due to thermal stresses and secures a suitable strength and stiffness. Many studies have examined FGM as thermal barriers. With the increased usage of these materials it is also important to understand the dynamics of FGM structures. A few studies have addressed this. Transient thermal stresses in a plate made of functionally gradient material were examined by Obata and Noda [5]. Vibration analysis of functionally graded cylindrical shells was performed by Loy et al. [3]. Recently, Lam et al. [4] presented dynamic stability analysis of functionally graded cylindrical shells under periodic axial loading. In this paper, the parametric vibrations or dynamic stability of functionally graded rectangular plate described by geometrically nonlinear partial differential equations is studied using the direct Liapunov method. Moderately large deflection 
equations taking into account a coupling of in-plane and transverse motions are used. Due to a small thickness coupling and rotary, inertia terms are neglected. Material properties are graded in the thickness direction of the plate according to volume friction power law distribution. The viscous model of external damping with a constant coefficient is assumed. An oscillating temperature causes generation of in-plane time-dependent forces destabilizing plane state of the plate equilibrium. The asymptotic stability and almost sure asymptotic stability criteria involving a damping coefficient and loading parameters are derived. Effects of power law exponent on the stability domains are studied.

\section{Problem formulation}

Consider the thin functionally graded rectangular plate with in-plane dimensions $a$ and $b$. In-plane and transverse displacements are denoted by $u, v$, and $w$, respectively. Taking into account the Kirchhoff hypothesis on nondeformable normal element and Kármántype geometric nonlinearity, the governing partial differential equations are given as follows (Whitney [8]):

$$
\begin{gathered}
N_{x, x}+N_{x y, y}=0, \\
N_{x y, x}+N_{y, y}=0, \\
w_{, t t}+2 d w_{, t}+\left(\bar{N}_{x}+\bar{N}_{0 x}\right) w_{, x x}+\left(\bar{N}_{x}+\bar{N}_{0 y}\right) w_{, y y}-M_{x, x x}-2 M_{x y, x y} \\
+-M_{y, y y}-N_{x} w_{, x x}-N_{y} w_{, y y}-2 N_{x y} w_{, x y}=0, \quad(x, y) \in \Omega \equiv(0, a) \times(0, b),
\end{gathered}
$$

where $d$ is a damping coefficient, $\bar{N}_{x}$ and $\bar{N}_{y}$ are time-dependent components of membrane forces, $\bar{N}_{0 x}$ and $\bar{N}_{0 y}$ are constant components of membrane forces divided by $\rho h$, $\rho$ is the equivalent density of the plate, $h$ is the total thickness. The membrane forces are stochastic with means equal to zero and known probability distributions. The processes are physically realizable and sufficiently smooth in order for the solution of dynamics equations to exist. We use the extensional, coupling, and bending stiffnesses $A_{i j}, B_{i j}$, and $D_{i j}(i, j)=1,2,6$ which are defined as follows:

$$
\left\{A_{i j}, B_{i j}, D_{i j}\right\}=\int_{-h / 2}^{h / 2} Q_{i j}\left\{1, z, z^{2}\right\} d z
$$

The reduced stiffnesses $Q_{i j}$, divided by $\rho h$, for isotropic materials are given by

$$
\mathbf{Q}=\frac{1}{\rho h}\left|\begin{array}{ccc}
\frac{E_{\mathrm{eff}}}{1-v_{\mathrm{eff}}^{2}} & \frac{\nu_{\mathrm{eff}} E_{\mathrm{eff}}}{1-\nu_{\mathrm{eff}}^{2}} & 0 \\
\frac{v_{\mathrm{eff}} E_{\mathrm{eff}}}{1-\nu_{\mathrm{eff}}^{2}} & \frac{E_{\mathrm{eff}}}{1-v_{\mathrm{eff}}^{2}} & 0 \\
0 & 0 & \frac{E_{\mathrm{eff}}}{2\left(1+\nu_{\mathrm{eff}}\right)}
\end{array}\right|
$$

Therefore matrix $\mathbf{Q}$ has the same form as the stiffness matrix of isotropic plate, but we should keep in mind its spatial dependency on $z$. In-plane and moments are expressed by 
displacements as follows:

$$
\left|\begin{array}{c}
N_{x} \\
N_{y} \\
N_{x y} \\
M_{x} \\
M_{y} \\
M_{x y}
\end{array}\right|=\left|\begin{array}{cccccc}
A_{11} & A_{12} & 0 & B_{11} & B_{12} & 0 \\
A_{12} & A_{22} & 0 & B_{12} & B_{11} & 0 \\
0 & 0 & A_{66} & 0 & 0 & B_{66} \\
B_{11} & B_{12} & 0 & D_{11} & D_{12} & 0 \\
B_{12} & B_{11} & 0 & D_{12} & D_{22} & 0 \\
0 & 0 & B_{66} & 0 & 0 & D_{66}
\end{array}\right|\left|\begin{array}{c}
u_{, x}+\frac{1}{2} w_{, x}^{2} \\
v_{, y}+\frac{1}{2} w_{, y}^{2} \\
u_{, y}+v_{, x}+w_{, x} w_{, y} \\
-w_{, x x} \\
-w_{, y y} \\
-2 w_{, x y}
\end{array}\right| .
$$

Using (2.5), the constitutive equation (2.6) can be rewritten in the form

$$
\begin{aligned}
\left|\begin{array}{c}
N_{x} \\
N_{y} \\
N_{x y} \\
M_{x} \\
M_{y} \\
M_{x y}
\end{array}\right| & \left|\begin{array}{cccccc}
A_{11} & A_{12} & 0 & B_{11} & B_{12} & 0 \\
A_{12} & A_{11} & 0 & B_{12} & B_{11} & 0 \\
0 & 0 & \frac{A_{11}-A_{12}}{2} & 0 & 0 & \frac{B_{11}-B_{12}}{2} \\
B_{11} & B_{12} & 0 & D_{11} & D_{12} & 0 \\
B_{12} & B_{11} & 0 & D_{12} & D_{11} & 0 \\
0 & 0 & \frac{B_{11}-B_{12}}{2} & 0 & 0 & \frac{D_{11}-D_{12}}{2}
\end{array}\right| \\
& \times\left|\begin{array}{c}
u_{, x}+\frac{1}{2} w_{, x}^{2} \\
v_{, y}+\frac{1}{2} w_{, y}^{2} \\
u_{, y}+v_{, x}+w_{, x} w_{, y} \\
-w_{, x x} \\
-w_{, y y} \\
-2 w_{, x y}
\end{array}\right| .
\end{aligned}
$$

The effective elastic modulus and the effective Poisson ratio of the functionally graded plate are denoted by $E_{\text {eff }}$ and $v_{\text {eff }}$, respectively. In order to model precisely the material properties of functionally graded materials, the properties must be both temperature and position dependent. This is achieved by using a rule of mixtures for the mechanical parameters $\left(E_{\text {eff }}, v_{\text {eff }}, \rho\right)$. The volume fraction is a spatial function and the properties of the constituents are functions of the temperature. The combination of these functions gives the effective material properties of functionally graded materials and can be expressed as follows:

$$
F_{\text {eff }}(T, z)=F_{c}(T) V(z)+F_{m}(T)(1-V(z)),
$$

where $F_{\text {eff }}$ is the effective material property of the functionally graded material, $F_{c}$ and $F_{m}$ are the properties of the ceramic and the metal, respectively, and $V$ is the volume fraction of the ceramic constituent of the functionally graded material. A simple power law exponent of the volume fractions is used to describe the amount of ceramic and metal 
in the functionally graded material as follows:

$$
V(z)=\left(\frac{z+h / 2}{h}\right)^{q}
$$

where $q$ is the power law exponent $(0 \leq q<\infty)$. The plate is assumed to be simply supported along each edge. The conditions imposed on displacements and internal forces and moments, called according to Almroth's [1] classifications S2, can be written down as

$$
\begin{aligned}
& w=0, \quad M_{x}=0, \quad N_{x}=0, \quad v=0, \quad \text { at } x=0, a, \\
& w=0, \quad M_{y}=0, \quad N_{y}=0, \quad u=0, \quad \text { at } y=0, b \text {. }
\end{aligned}
$$

It is assumed that the plate is subjected to the time-varying in-plane axial forces $\bar{N}_{x}$ and $\bar{N}_{y}$ leading to parametric vibrations. The transverse motion of the plate is described by the nonlinear uniform equations (2.1), (2.2), and (2.3) with the trivial solution $w=0$ corresponding to the plane (undisturbed) state. The trivial solution is called almost sure asymptotically stable if

$$
P\left\{\lim _{t \rightarrow \infty}\|w(\cdot, t)\|=0\right\}=1,
$$

where $\|w(\cdot, t)\|$ is a measure of disturbed solution $w$ from the equilibrium state, and $P$ is a probability measure. The crucial point of the method is a construction of a suitable Liapunov functional, which is positive for any motion of the analyzed system. It follows that the measure of distance can be chosen as the square root of Liapunov functional $\|w(\cdot, t)\|=V^{1 / 2}$.

\section{Stability analysis}

The energy-like Liapunov functional has the form of a sum of modified kinetic energy $\mathscr{T}$ and potential energy of the plate, and can be chosen in the form similar to the functional involved in stability analysis of laminated plates (Tylikowski [6]):

$$
\begin{aligned}
\mathscr{V}_{n}=\mathscr{T}+\frac{1}{2} \int_{\Omega}[ & -M_{x} w_{, x x}-M_{y} w_{, y y}-2 M_{x y} w_{, x y}+N_{x}\left(u_{, x}+\frac{1}{2} w_{, x}^{2}\right) \\
& +N_{y}\left(v_{, y}+\frac{1}{2} w_{, y}^{2}\right)+N_{x y}\left(u_{, y}+v_{, x}+w_{, x} w_{, y}\right) \\
& \left.-\bar{N}_{0 x} w_{, x}^{2}-\bar{N}_{0 y} w_{, y}^{2}\right] d \Omega,
\end{aligned}
$$

where $\mathscr{T}$ expressed by $w$ and the transverse velocity $v=w_{, t}$ is given by

$$
\mathscr{T}=\frac{1}{2} \int_{\Omega}\left(v^{2}+2 d v w+2 d^{2} w^{2}\right) d \Omega
$$


It may be observed that contrary to the linear or linearized case, $\mathscr{V}_{n}$ is the fourth-order functional. Substituting constitutive equation (2.6), we have

$$
\begin{aligned}
\mathscr{V}_{n}=\mathcal{T}+\frac{1}{2} \int_{\Omega}[ & A_{11}\left(u_{, x}+\frac{1}{2} w_{, x}^{2}\right)^{2}+2 A_{12}\left(u_{, x}+\frac{1}{2} w_{, x}^{2}\right)\left(v_{, y}+\frac{1}{2} w_{, y}^{2}\right) \\
& +A_{22}\left(v_{, y}+\frac{1}{2} w_{, y}^{2}\right)^{2}+A_{66}\left(u_{, y}+v_{, x}+w_{, x} w_{, y}\right)^{2} \\
& -2 B_{11}\left(u_{, x}+\frac{1}{2} w_{, x}^{2}\right) w_{, x x}-2 B_{12}\left(u_{, x}+\frac{1}{2} w_{, x}^{2}\right) w_{, y y} \\
& -2 B_{12}\left(v_{, y}+\frac{1}{2} w_{, y}^{2}\right) w_{, x x}-2 B_{11}\left(v_{, y}+\frac{1}{2} w_{, y}^{2}\right) w_{, y y} \\
& -4 B_{66}\left(u_{, y}+v_{, x}+w_{, x} w_{, y}\right) w_{x y}+D_{11} w_{, x x}^{2} \\
& \left.+2 D_{12} w_{, x x} w_{, y y}+D_{11} w_{, y y}^{2}+4 D_{66} w_{, x y}^{2}-\bar{N}_{0 x} w_{, x}^{2}-\bar{N}_{0 y} w_{, y}^{2}\right] d \Omega .
\end{aligned}
$$

It is assumed that the in-plane forces are periodic or a stochastic nonwhite stationary and sufficiently smooth ergodic process. Therefore, it is legitimate to use the classical differentiation rule. Upon differentiation with respect to time, substituting dynamic equations (2.1), (2.2), and (2.3) and using the boundary conditions, we obtain the time derivative of functional in the form

$$
\frac{d \mathscr{V}_{n}}{d t}=-2 \lambda \mathscr{V}_{n}+2 \mathcal{U}_{n}
$$

where the auxiliary functional $u_{n}$ is defined as follows:

$$
\begin{gathered}
u_{n}=u-\frac{d}{2} \int_{\Omega}\left[N_{x}\left(u_{, x}+\frac{1}{2} w_{, x}^{2}\right)+N_{y}\left(v_{, y}+\frac{1}{2} w_{, y}^{2}\right)+N_{x y}\left(u_{, y}+v_{, x}+w_{, x} w_{, y}\right)\right] d \Omega, \\
u=\frac{1}{2} \int_{\Omega}\left[2 d^{2} w w_{, t}+2 d^{3} w^{2}+\left(w_{, t}+d w\right)\left(\bar{N}_{x} w_{, x x}+\bar{N}_{y} w_{, y y}\right)\right] d \Omega .
\end{gathered}
$$

Eliminating in-plane forces by means of (2.7) we have

$$
\begin{aligned}
u_{n}=u-\frac{d}{2} \int_{\Omega}[ & A_{11}\left(u_{, x}+\frac{1}{2} w_{, x}^{2}\right)^{2}+2 A_{12}\left(u_{, x}+\frac{1}{2} w_{, x}^{2}\right)\left(v_{, y}+\frac{1}{2} w_{, y}^{2}\right) \\
& +A_{22}\left(v_{, y}+\frac{1}{2} w_{, y}^{2}\right)^{2}+A_{66}\left(u_{, y}+v_{, x}+w_{, x} w_{, y}\right)^{2} \\
& -B_{11}\left(u_{, x}+\frac{1}{2} w_{, x}^{2}\right) w_{, x x}-B_{12}\left(u_{, x}+\frac{1}{2} w_{, x}^{2}\right) w_{, y y} \\
& -B_{12}\left(v_{, y}+\frac{1}{2} w_{, y}^{2}\right) w_{, x x}-B_{11}\left(v_{, y}+\frac{1}{2} w_{, y}^{2}\right) w_{, y y} \\
& \left.-2 B_{66}\left(u_{, y}+v_{, x}+w_{, x} w_{, y}\right) w_{x y}\right] d \Omega .
\end{aligned}
$$


Therefore, the stability analysis of the nonlinear system depends on the construction of the bound

$$
u_{n} \leq \lambda \mathscr{V}_{n}
$$

or we look for a function $\lambda$ defined as a maximum over all admissible functions $u, v, w$, $w_{, t}$ and satisfying the boundary conditions of the ratio $\mathcal{U}_{n} / \mathscr{V}_{n}$. As a maximum is a particular case of stationary point, we put to zero a variation of $\mathfrak{U}_{n} / \mathscr{V}_{n}$. The associate Euler equations are nonlinear in the case of the fourth-order functionals. It complicates a stability analysis and in order to obtain the analytical form of function $\lambda$, we have to modify the variational problem. Therefore, our object is to find such second-order functionals $V^{*}$ and $U^{*}$ that the inequality

$$
U^{*} \leq \lambda V^{*}
$$

will make inequality (3.8) be true. In order to do this we express functional (3.1) in the form

$$
\begin{aligned}
\mathscr{V}_{n}=V+V_{p}-\frac{1}{2} \int_{\Omega} & {\left[D_{11}\left(\frac{w_{, x x}}{2 k}\right)^{2}+2 D_{12} \frac{w_{, x x}}{2 k} \frac{w_{, y y}}{2 k}+D_{22}\left(\frac{w_{, y y}}{2 k}\right)^{2}\right.} \\
& +4 D_{66}\left(\frac{w_{, x y}}{2 k}\right)^{2}-B_{11}\left(u_{, x} w_{, x x}+v_{, y} w_{, y y}\right) \\
& \left.-B_{12}\left(u_{, x} w_{, y y}+v_{, y} w_{, x x}\right)-2 B_{66} w_{, x y}\left(u_{, y}+v_{, x}\right)\right] d \Omega,
\end{aligned}
$$

where $V$ is the second-order Liapunov functional for a linearized problem

$$
\begin{aligned}
V=\mathscr{T}+\frac{1}{2} \int_{\Omega}[ & D_{11} w_{, x x}^{2}+2 D_{12} w_{, x x} w_{, y y}+D_{22} w_{, y y}^{2} \\
& +4 D_{66} w_{, x y}^{2}-B_{11}\left(u_{, x} w_{, x x}+v_{, y} w_{, y y}\right) \\
& -B_{12}\left(u_{, x} w_{, y y}+v_{, y} w_{, x x}\right)-2 B_{66} w_{, x y}\left(u_{, y}+v_{, x}\right) \\
& \left.-\bar{N}_{0 x} w_{, x}^{2}-\bar{N}_{0 y} w_{, y}^{2}\right] d \Omega .
\end{aligned}
$$

$V_{p}$ is a positive definite fourth-order functional

$$
V_{p}=\frac{1}{2} \int_{\Omega} \mathbf{z}^{T} \mathbf{C z} d \Omega,
$$


and $k$ is a number greater than 1 chosen so that we will obtain the greatest stability region. The matrix $\mathbf{C}$ is given as follows:

$$
\mathbf{C}(k)=\left|\begin{array}{cccccc}
A_{11} & A_{12} & 0 & 2 k B_{11} & 2 k B_{12} & 0 \\
A_{12} & A_{11} & 0 & 2 k B_{12} & 2 k B_{11} & 0 \\
0 & 0 & \frac{A_{11}-A_{12}}{2} & 0 & 0 & \frac{2 k\left(B_{11}-B_{12}\right)}{2} \\
2 k B_{11} & 2 k B_{12} & 0 & D_{11} & D_{12} & 0 \\
2 k B_{12} & 2 k B_{11} & 0 & D_{12} & D_{11} & 0 \\
0 & 0 & \frac{2 k\left(B_{11}-B_{12}\right)}{2} & 0 & 0 & \frac{D_{11}-D_{12}}{2}
\end{array}\right|,
$$

where $(\cdot)^{T}$ denotes a transposition of matrix and the $\mathbf{z}$ is a modified state of strain defined by a column matrix

$$
\mathbf{z}=\left|\begin{array}{c}
u_{, x}+\frac{1}{2} w_{, x}^{2} \\
v_{, y}+\frac{1}{2} w_{, y}^{2} \\
u_{, y}+v_{, x}+w_{, x} w_{, y} \\
-\frac{w_{, x x}}{2 k} \\
-\frac{w_{, y y}}{2 k} \\
-\frac{2 w_{, x y}}{2 k}
\end{array}\right|
$$

The functional $V_{p}$ is positive definite if the Sylvester conditions of positive definiteness for matrix $\mathbf{C}$ are satisfied (see Gantmacher [2]):

$$
\operatorname{det}\left|\begin{array}{cccccc}
A_{11} & A_{12} & 0 & 2 k B_{11} & 2 k B_{12} & 0 \\
A_{12} & A_{11} & 0 & 2 k B_{12} & 2 k B_{11} & 0 \\
0 & 0 & \frac{A_{11}-A_{12}}{2} & 0 & 0 & \frac{2 k\left(B_{11}-B_{12}\right)}{2} \\
2 k B_{11} & 2 k B_{12} & 0 & D_{11} & D_{12} & 0 \\
2 k B_{12} & 2 k B_{11} & 0 & D_{12} & D_{11} & 0 \\
0 & 0 & \frac{k\left(B_{11}-B_{12}\right)}{2} & 0 & 0 & \frac{D_{11}-D_{12}}{2}
\end{array}\right|>0
$$


Dynamic stability of functionally graded plate

$\operatorname{det}\left|\begin{array}{ccccc}A_{11} & A_{12} & 0 & 2 k B_{11} & 2 k B_{12} \\ A_{12} & A_{11} & 0 & 2 k B_{12} & 2 k B_{11} \\ 0 & 0 & \frac{A_{11}-A_{12}}{2} & 0 & 0 \\ 2 k B_{11} & 2 k B_{12} & 0 & D_{11} & D_{12} \\ 2 k B_{12} & 2 k B_{11} & 0 & D_{12} & D_{11}\end{array}\right|>0$

$$
\operatorname{det}\left|\begin{array}{cccc}
A_{11} & A_{12} & 0 & 2 k B_{11} \\
A_{12} & A_{11} & 0 & 2 k B_{12} \\
0 & 0 & \frac{A_{11}-A_{12}}{2} & 0 \\
2 k B_{11} & 2 k B_{12} & 0 & D_{11}
\end{array}\right|>0 .
$$

Solving Sylvester's inequalities we obtain the number $k$ as a function of exponent $q$. Omitting the fourth-order functional $V_{p}$, we obtain the lower estimation of functional $\mathscr{V}_{n}$ by the second-order functional

$$
\mathscr{V}_{n} \geq V^{*}=V-\frac{1}{4 k^{2}} V_{b},
$$

where

$$
V_{b}=\frac{1}{2} \int_{\Omega}\left[D_{11} w_{, x x}^{2}+2 D_{12} w_{, x x} w_{, y y}+D_{22} w_{, y y}^{2}+4 D_{66} w_{, x y}^{2}\right] d \Omega
$$

In the way similar to derivation of estimation (3.18), we can rewrite (3.6) in the form

$$
\begin{aligned}
& u_{n}=u-\frac{d}{2} \int_{\Omega}[ A_{11}\left(u_{, x}+\frac{1}{2} w_{, x}^{2}\right)^{2}+2 A_{12}\left(u_{, x}+\frac{1}{2} w_{, x}^{2}\right)\left(v_{, y}+\frac{1}{2} w_{, y}^{2}\right) \\
&+A_{22}\left(v_{, y}+\frac{1}{2} w_{, y}^{2}\right)^{2}+A_{66}\left(u_{, y}+v_{, x}+w_{, x} w_{, y}\right)^{2} \\
&-4 k B_{11}\left(u_{, x}+\frac{1}{2} w_{, x}^{2}\right)\left(\frac{w_{, x x}}{4 k}\right)-4 k B_{12}\left(u_{, x}+\frac{1}{2} w_{, x}^{2}\right)\left(\frac{w_{, y y}}{4 k}\right) \\
&-4 k B_{12}\left(v_{, y}+\frac{1}{2} w_{, y}^{2}\right)\left(\frac{w_{, x x}}{4 k}\right)-4 k B_{11}\left(v_{, y}+\frac{1}{2} w_{, y}^{2}\right)\left(\frac{w, y y}{4 k}\right) \\
&-8 k B_{66}\left(u, y+v_{, x}+w_{, x} w_{, y}\right)\left(\frac{w_{x y}}{4 k}\right)+D_{11}\left(\frac{w_{, x x}}{4 k}\right)^{2} \\
&+2 D_{12}\left(\frac{w_{, x x}}{4 k}\right)\left(\frac{w_{, y y}}{4 k}\right)+D_{22}\left(\frac{w_{, y y}}{4 k}\right)^{2} \\
&\left.+4 D_{66}\left(\frac{w_{, x y}}{4 k}\right)^{2}\right] d \Omega \\
&+\frac{d}{32 k^{2}} \int_{\Omega}\left[D_{11} w_{, x x}^{2}+2 D_{12} w_{, x x} w_{, y y}+D_{22} w_{, y y}^{2}+4 D_{66} w_{, x y}^{2}\right] d \Omega .
\end{aligned}
$$


Omitting as negative the second integral, we have upper estimation of functional $u_{n}$

$$
u_{n} \leq U+\frac{d}{16 k^{2}} V_{b}
$$

Now we see that if a function $\lambda$ satisfies the following condition for the second-order functionals:

$$
U+\frac{d}{16 k^{2}} V_{b} \leq \lambda\left(V-\frac{1}{4 k^{2}} V_{b}\right)
$$

then the same function $\lambda$ will satisfy inequality (3.8).

Solving the associated Euler problem, we find the function $\lambda$ as follows:

$$
\begin{aligned}
\lambda=\max _{i, j=1,2, \ldots}\{ & \left\{\frac{d}{4 k^{2}} \Omega_{i j}^{2}\right. \\
& \left.+\sqrt{\frac{d^{2}}{16 k^{4}} \Omega_{i j}^{4}+4\left[4 d^{2}+\left(4-\frac{1}{k^{2}}\right) \Omega_{i j}^{2}+4 \kappa_{i j}\right]\left[2 d^{2}+\bar{N}_{x}\left(\frac{i \pi}{a}\right)^{2}+\bar{N}_{y}\left(\frac{j \pi}{b}\right)^{2}\right]^{2}}\right\} \\
& \left.\times\left(2\left[4 d^{2}+\left(4-\frac{1}{k^{2}}\right) \Omega_{i j}^{2}+4 \kappa_{i j}\right]\right)^{-1}\right\},
\end{aligned}
$$

where $\Omega_{i j}$ denotes the eigen frequency of the plate without couplings between in-plane and bending effects:

$$
\Omega_{i j}^{2}=D_{11}\left(\frac{i \pi}{a}\right)^{4}+2\left(D_{12}+2 D_{66}\right)\left(\frac{i \pi}{a}\right)^{2}\left(\frac{j \pi}{b}\right)^{2}+D_{22}\left(\frac{j \pi}{b}\right)^{4} .
$$

The bending-extension coupling and the influence of constant forces $\bar{N}_{0 x}$ and $\bar{N}_{0 y}$ are represented by $\kappa_{i j}$ :

$$
\begin{aligned}
\kappa_{i j}= & -B_{11} \frac{\left[\left(\frac{i \pi}{a}\right)^{2}+\left(\frac{j \pi}{b}\right)^{2}\right]^{2}\left[\alpha_{22}\left(\frac{i \pi}{a}\right)^{2}-2 \alpha_{12} \frac{i \pi}{a} \frac{j \pi}{b}+\alpha_{11}\left(\frac{j \pi}{b}\right)^{2}\right]}{\alpha_{11} \alpha_{22}-\alpha_{12}^{2}} \\
& -\bar{N}_{0 x}\left(\frac{i \pi}{a}\right)^{2}-\bar{N}_{0 y}\left(\frac{j \pi}{b}\right)^{2},
\end{aligned}
$$

where

$$
\begin{gathered}
\alpha_{11}=A_{11}\left(\frac{i \pi}{a}\right)^{2}+\frac{A_{11}-A_{12}}{2}\left(\frac{j \pi}{b}\right)^{2}, \quad \alpha_{12}=\frac{A_{11}+A_{12}}{2} \frac{i \pi}{a} \frac{j \pi}{b}, \\
\alpha_{22}=A_{11}\left(\frac{j \pi}{b}\right)^{2}+\frac{A_{11}-A_{12}}{2}\left(\frac{i \pi}{a}\right)^{2} .
\end{gathered}
$$


Table 4.1. Mechanical properties of constituents of the FGM.

\begin{tabular}{l|cc|cc|cc}
\hline \multicolumn{2}{c|}{ Material } & \multicolumn{2}{|c|}{ Steel-zirconia } & \multicolumn{2}{c|}{ Nickel-SiN } & \multicolumn{2}{c}{ Aluminum-TiC } \\
\hline$\rho, \quad \mathrm{kg} / \mathrm{m}^{3}$ & 8166 & 5700 & 8900 & 2370 & 2700 & 4920 \\
$E, \quad \mathrm{~N} / \mathrm{m}^{2}$ & $2.01 \times 10^{11}$ & $2.44 \times 10^{11}$ & $2.24 \times 10^{11}$ & $3.48 \times 10^{11}$ & $0.69 \times 10^{11}$ & $4.80 \times 10^{11}$ \\
$\nu$ & 0.33 & 0.288 & 0.31 & 0.24 & 0.33 & 0.2 \\
\hline
\end{tabular}

Table 4.2. Numbers $k_{\max }$ for crucial stability condition (3.22).

\begin{tabular}{cccccc}
\hline$q$ & 0.5 & 1 & 5 & 10 & 100 \\
\hline Steel-zirconia & 9.85 & 7.69 & 10.23 & 15.79 & 119.75 \\
Nickel-SiN & 4.70 & 3.56 & 4.40 & 6.63 & 48.22 \\
Aluminum-TiC & 1.65 & 1.10 & 0.912 & 1.123 & 5.23 \\
\hline
\end{tabular}

Using the property of function $\lambda$ in equality (3.2) leads to the first-order differential inequality, the solution of which has the form

$$
\mathscr{V}_{n}(t) \leq \mathscr{V}_{n}(0) \exp \left[-\left(d-\frac{1}{t} \int_{0}^{t} \lambda(\tau) d \tau\right) t\right]
$$

Therefore, the sufficient criterion of the asymptotic stability has the form

$$
d \geq \lim _{t \rightarrow \infty} \frac{1}{t} \int_{0}^{t} \lambda(\tau) d \tau
$$

If the processes $\bar{N}_{x}$ and $\bar{N}_{y}$ satisfy an ergodic property, the sufficient condition of the almost sure asymptotic stability can be written as follows:

$$
d \geq \mathbf{E} \lambda
$$

where $\mathbf{E}$ denotes the mathematical expectation.

\section{Numerical results}

The functionally graded materials used in this study are steel-zirconia, nickel-silicon nitride, and aluminum-titanium carbide. Mechanical properties are given in Table 4.1.

The plate dimensions are as follows: $h=0.005 \mathrm{~m}, a=b=0.5 \mathrm{~m}$. The number $k$ maximizing stability region calculated from the Sylvester inequalities is given in Table 4.2 for $q=0.5,1,5,10,100$. Formulae (3.23) and (3.29) give us the possibility to calculate a maximal excitation intensity (e.g., square root of variance) of modified in-plane forces $\left(\bar{N}_{x} / \rho h\right.$ $\left.\left[\mathrm{m}^{2} / \mathrm{s}^{2}\right], \bar{N}_{y} / \rho h\left[\mathrm{~m}^{2} / \mathrm{s}^{2}\right]\right)$ guaranteeing the almost sure asymptotic stability for given values of power law exponent $q$. The stability regions are calculated for Gaussian and harmonic forces with variance $s^{2}\left[\mathrm{~m}^{4} / \mathrm{s}^{4}\right]$ and $d[1 / s]$. Stability domain boundaries of FGM plate, are shown in Figures 4.1, 4.2, 4.3, and 4.5 for the following exponents: $q=0.5, q=1, q=5$, $q=10$, and $q=100$. Stability domain boundaries of FGM plate for the different values of in-plane force are shown in Figure 4.4. 


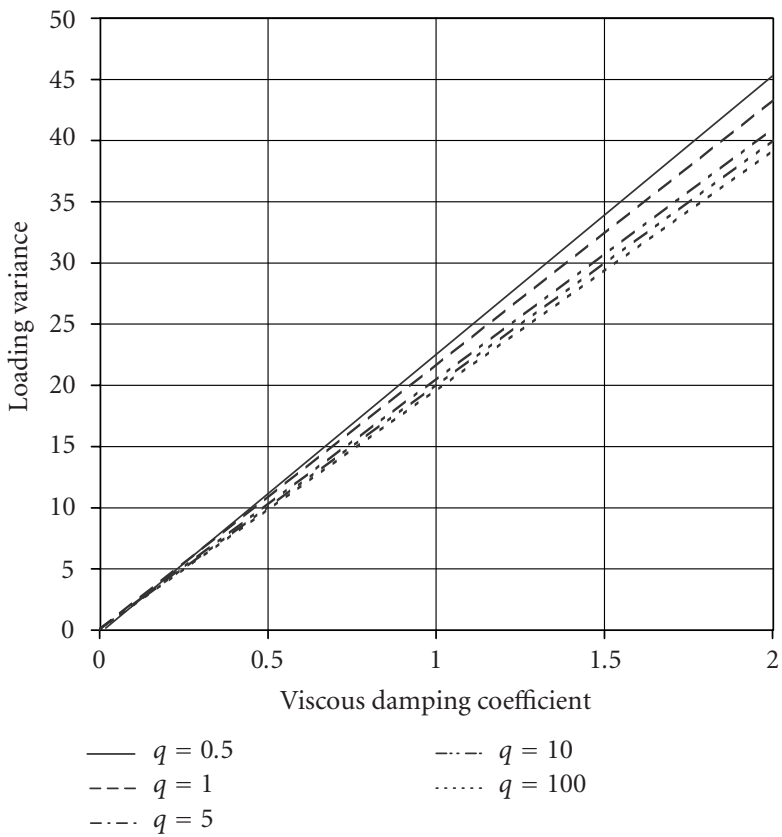

Figure 4.1. Influence of the power law exponent on stability domains for the steel-zirconia FGM.

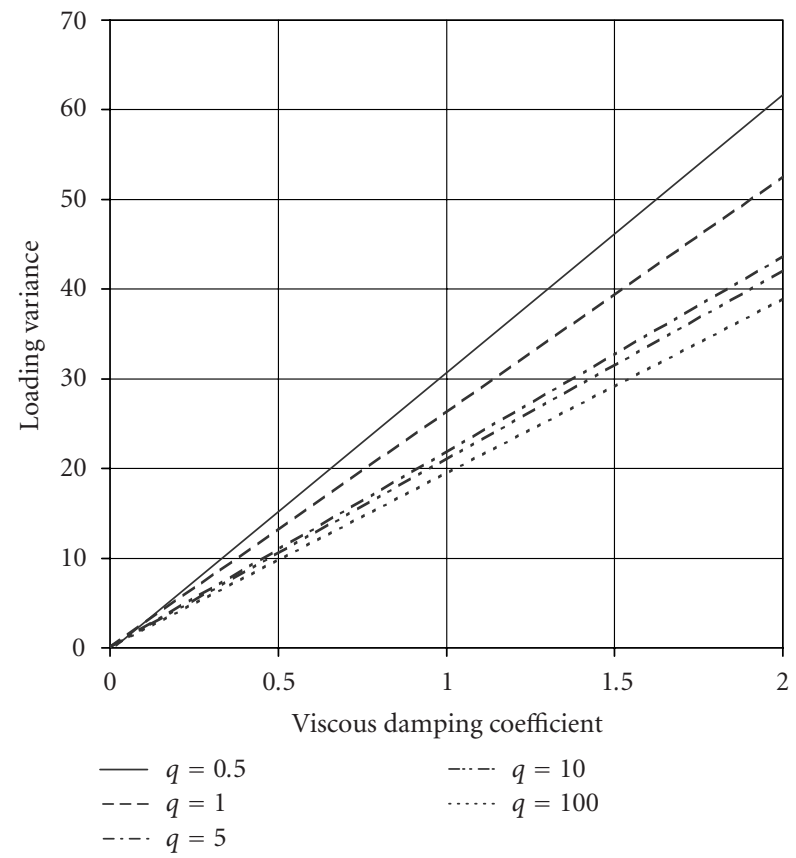

Figure 4.2. Influence of the power law exponent on stability domains for the nickel-SiN FGM. 


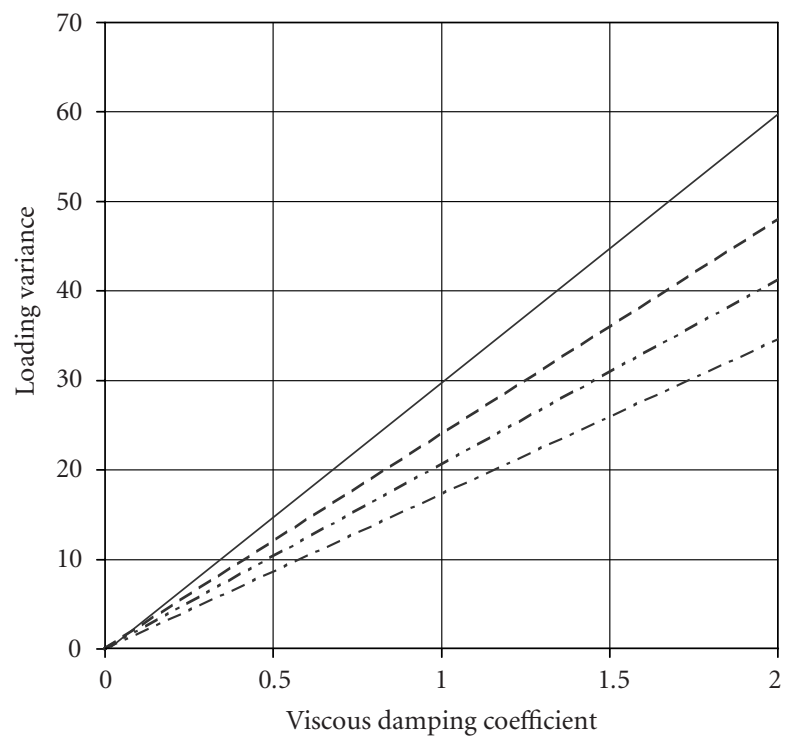

$$
\begin{aligned}
& -q=0.5 \\
& q=1 \\
& \text {-.. } q=5 \\
& \text {-.- } q=10
\end{aligned}
$$

Figure 4.3. Influence of the power law exponent on stability domains for the aluminum-TiC FGM.

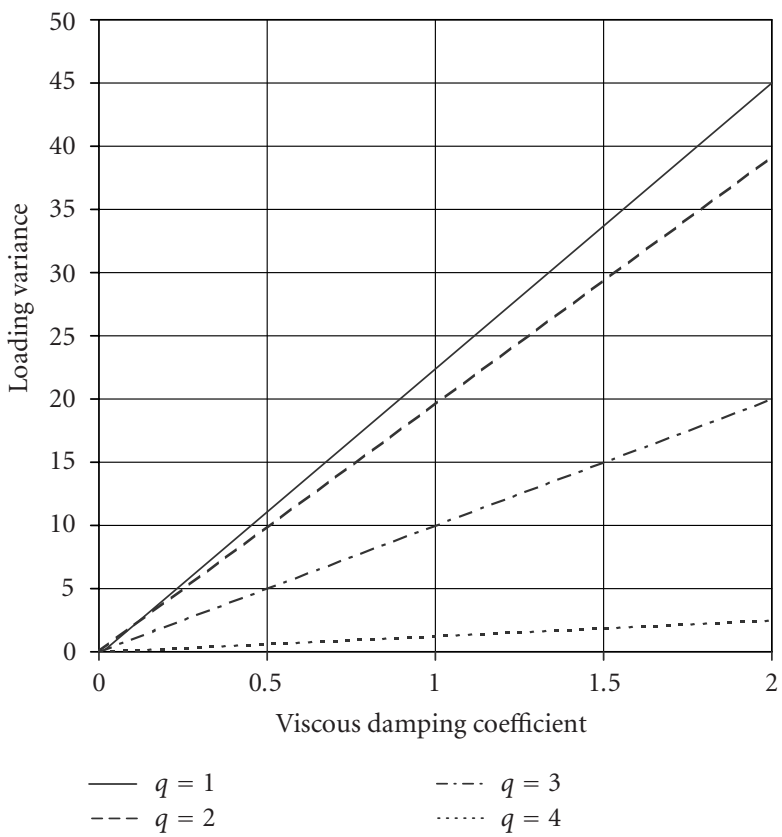

Figure 4.4. Influence of the constant component of in-plane force on stability domains for the steelzirconia FGM ( $q=0.5):(1) \bar{N}_{0 x}=0$, (2) $\bar{N}_{0 x}=10000$, (3) $\bar{N}_{0 x}=12000$, and (4) $\bar{N}_{0 x}=12600$. 


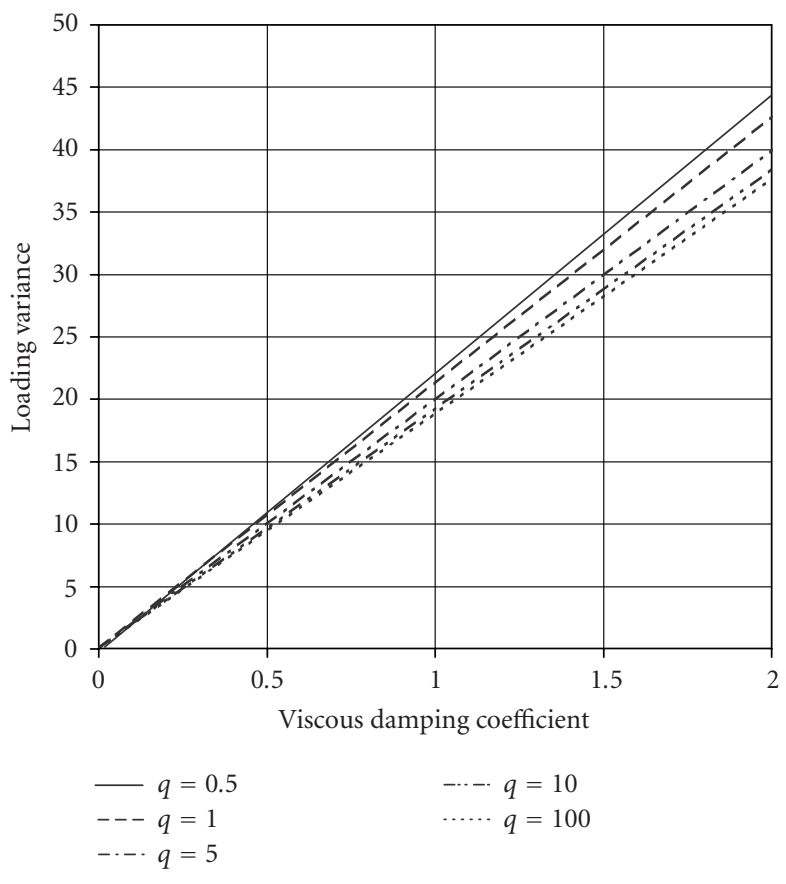

Figure 4.5. Influence of the power law exponent on stability domains for the steel-zirconia FGM.

\section{Conclusions}

The applicability of the direct Liapunov method has been extended to geometrically nonlinear functionally graded plates subjected to time-dependent, in-plane forces. The major conclusion is that the linearized problem should be modified to ensure the stability of nonlinear problem. The influence of the power law exponent and the constant component of in-plane force on the critical value of stability domains (expressed by the variance of time-dependent force component) is shown. Stability domains depend essentially on the constant compressive force. The influence of probability distribution on stability domains is merely noticeable.

\section{References}

[1] B. O. Almroth, Influence of edge conditions on the stability of axially compressed cylindrical shells, AIAA J. 4 (1966), 134-140.

[2] F. R. Gantmacher, The Theory of Matrices. Vols. 1, 2, Chelsea Publishing, New York, 1959.

[3] C. T. Loy, K. Y. Lam, and J. N. Reddy, Vibration of functionally graded cylindrical shells, Int. J. Mech. Sci. 41 (1999), no. 3, 309-324.

[4] T. Y. Ng, K. Y. Lam, K. M. Liew, and J. N. Reddy, Dynamic stability analysis of functionally graded cylindrical shells under periodic axial loading, Internat. J. Solids Structures 38 (2001), no. 8, 1295-1309.

[5] Y. Obata and N. Noda, Transient thermal stresses in a plate of functionally gradient material, Functionally Gradient Materials (J. B. Holt, et al., eds.), Ceram. Trans., vol. 34, American Ceramic Society, Ohio, 1993, pp. 403-410. 


\section{Dynamic stability of functionally graded plate}

[6] A. Tylikowski, Dynamic stability of nonlinear antisymmetrically-laminated cross-ply rectangular plates, ASME J. Appl. Mech. 56 (1989), 375-381.

[7] Stability of functionally graded plate under in-plane time-dependent compression, Int. J. Mech. Mech. Eng. 7 (2004), no. 2, 5-12.

[8] J. M. Whitney, Structural Analysis of Laminated Anisotropic Plates, chapter 2.7, Technomic Publishing, Lancaster, 1987.

Andrzej Tylikowski: Warsaw University of Technology, Narbutta 84, 02-524 Warszawa, Poland E-mail address: aty@simr.pw.edu.pl 


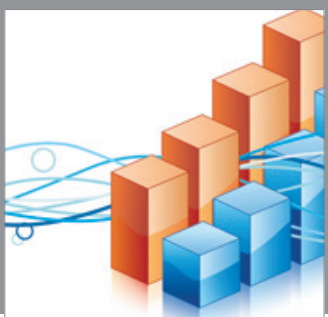

Advances in

Operations Research

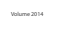

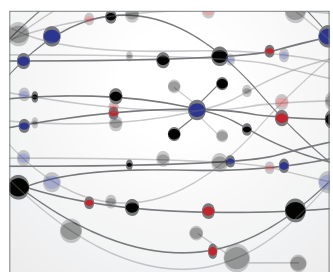

\section{The Scientific} World Journal
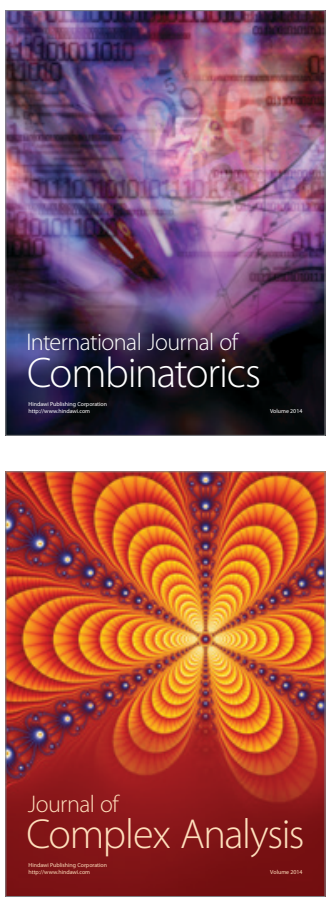

International Journal of

Mathematics and

Mathematical

Sciences
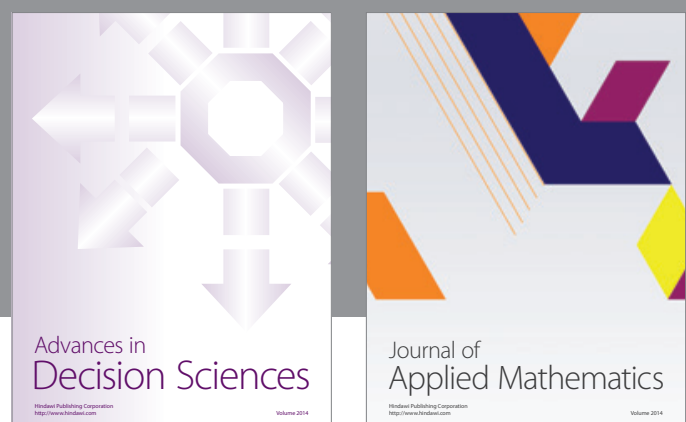

Journal of

Applied Mathematics
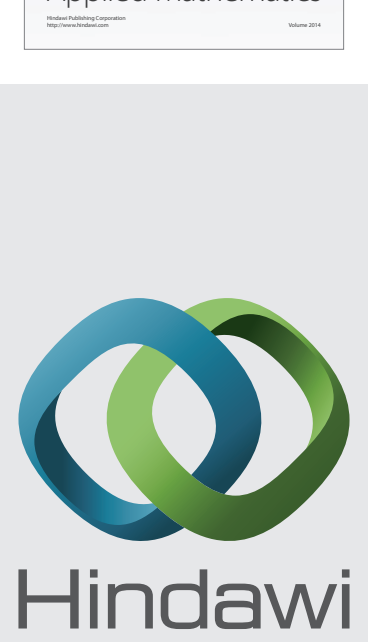

Submit your manuscripts at http://www.hindawi.com
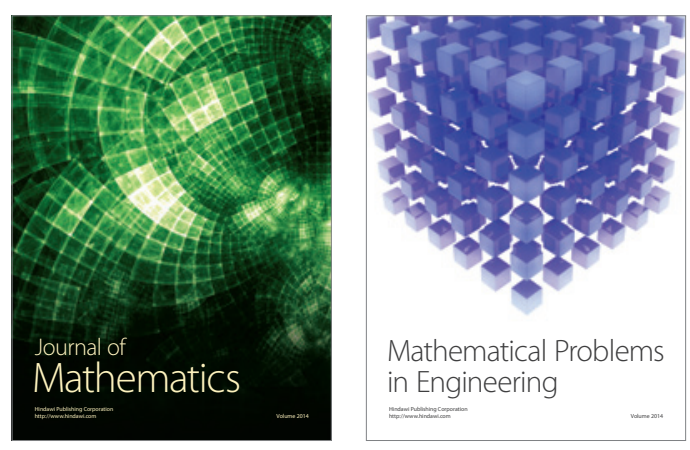

Mathematical Problems in Engineering
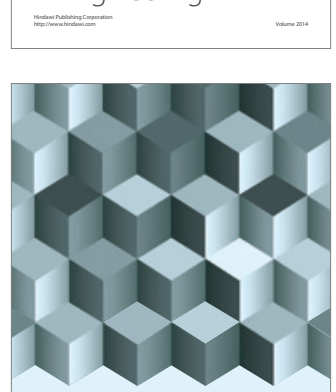

Journal of

Function Spaces
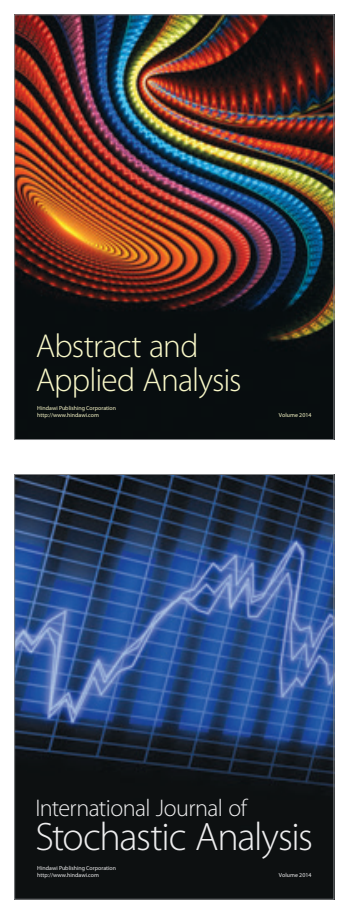

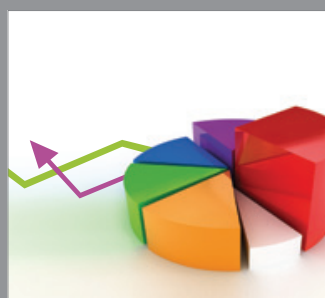

ournal of

Probability and Statistics

Promensencen
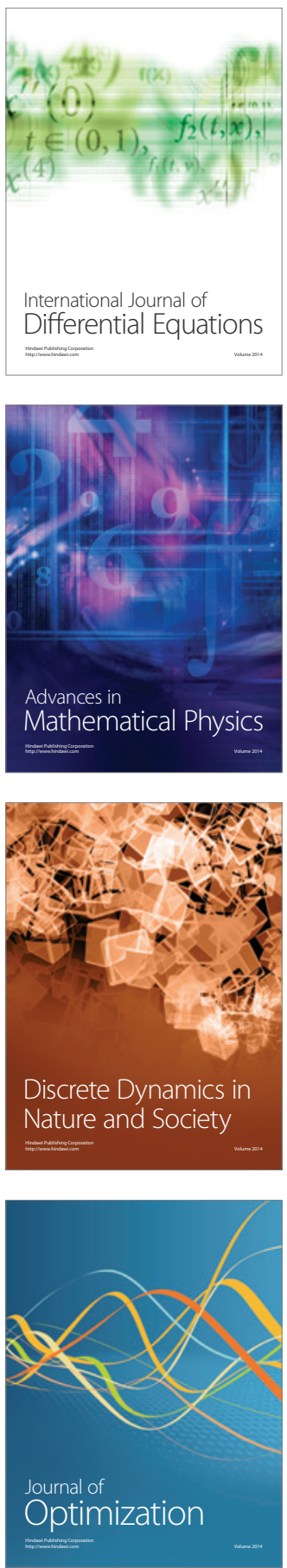